\title{
Development and Validation of a RANS-based Airdrop Simulation Approach
}

\author{
Sven Geisbauer* \\ DLR, German Aerospace Center, 38108 Braunschweig, Germany \\ and \\ Hauke Schmidt ${ }^{\dagger}$ \\ DLR, German Aerospace Center, 37073 Göttingen, Germany
}

\begin{abstract}
During the first few seconds of an airdrop the payload interacts with the flow field behind the aircraft. As most airdrop simulation tools rely on simplified aerodynamic models, e.g. ones with frozen wind field information, DLR has developed an alternative simulation approach. It computes the aerodynamic interference effects during the initial phase of an airdrop and the resulting trajectory. To achieve this the DLR TAU flow solver has been coupled with the multi-body simulation software SIMPACK. The major advantage of this approach, apart from the fact that it does not rely on simplified aerodynamic models, is its multi-body functionality. Thus, the relative motion of bodies, for example between payload and parachute, can easily be examined as kinematic constraints are considered. DLR has successfully demonstrated that its airdrop simulation approach is well-suited to predict the trajectories of different cargo and cargo-parachute configurations. An overview on the methodology, the verification and validation of this approach is presented in the following.
\end{abstract}

\section{Introduction}

Ardrop operations belong to the standard repertoire of modern military transport aircraft. The deA ployment of personnel, military equipment or humanitarian aid supplies is an agile and flexible way of globally providing support in conflict areas or disaster zones. To safely conduct these operations it needs to be ensured that the behavior of the supply which is to be airdropped is known in advance. For example, very lightweight or aerodynamically sensitive supplies are more susceptible to interact with the wake of the aircraft than large and heavy cargo pallets. During the deployment of the latter, however, a significant pitch-up motion of the aircraft might occur due to the shift of the center of gravity. If such a deployment is carried out at low altitudes it is obvious that there is no room for mistakes. The ability to accurately predict the attitude and trajectory of an airdropped supply, as well as its interference with the aircraft, therefore is essential to get approval for airdrop operations.

Traditionally, the development and certification of airdrop systems has been a time-consuming and costly process several instrumented flight tests are usually involved. These are necessary to demonstrate that the behavior of a dropped supply meets the specifications and certification requirements. To aid designing new precision airdrop systems and to gradually reduce the high unit costs by minimizing the number of instrumented flight tests required there has been an increasing demand to provide accurate and well-validated simulation tools. ${ }^{1}$

\footnotetext{
${ }^{*}$ Research Scientist, Institute of Aerodynamics and Flow Technology, Lilienthalplatz 7, AIAA member.

${ }^{\dagger}$ Research Scientist, formerly Institute of Aeroelasticity, Bunsenstraße 10.
} 
Maturing simulation methods up to the point where a complete airdrop sequence, from leaving the aircraft until landing, can be virtually predicted with guaranteed accuracy is a very challenging task. Especially the aerodynamic forces and moments need to be properly modeled to achieve reasonable results. Therefore, many research groups focused on investigating the aerodynamic properties of the wake ${ }^{2-5}$ of military transport aircrafts or of airdrop supplies, ${ }^{6-8}$ either by applying numerical simulations or experiments. They basically proved that the flow field behind a common military transport aircraft could be accurately simulated using detached-eddy simulation methods. However, these methods have not yet found their way into an integrated simulation approach which is capable of predicting the time-dependent, 6-degree of freedom (DOF) motion of a dropped supply under full consideration of the aerodynamic effects. For example, although more recent approaches to simulate store separations do consider a free 6-DOF motion the aerodynamics still is assumed to be inviscid. ${ }^{9,10}$

To serve the general demand for well-validated simulation tools DLR has begun in 2006 to extend its competence in assessing airdrop operations within the internal projects MiTraPor ${ }^{a}$ and MiTraPor 2. The DLR Institutes of Aerodynamics and Flow Technology, Aeroelasticity and Flight Systems closely collaborated to establish a simulation approach which enables DLR to accurately predict the trajectory and time-dependent motion of an airdropped supply, from store release until landing. ${ }^{11}$ A multi-fidelity simulation approach was established, which on the one hand allows for the real-time computation of entire trajectories based on aerodynamic lookup tables, including the influence of different types and numbers of attached parachute systems. $^{12,13}$ The strong interference effects between the wake of the aircraft and the initial motion of the dropped supply, however, cannot be examined with this approach. Hence, a high-fidelity simulation ap-

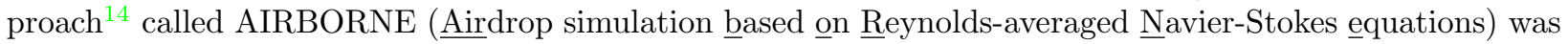
developed simultaneously. It combines state-of-the-art CFD methods with a multi-body simulation tool and enables DLR to investigate the initial trajectory of a dropped supply under the aerodynamic influence of the aircraft.

In contrast to the conclusions of the aforementioned research groups DLR has decided to stick with its Reynolds-averaged Navier-Stokes flow solver TAU to examine and predict the airdrop trajectories. Based on a vast experimental data base, including PIV flow field ${ }^{15,16}$ and trajectory measurements, ${ }^{17}$ the suitability of the DLR TAU code to predict the general flow field ${ }^{18,19}$ as well as first trajectories ${ }^{20}$ was successfully demonstrated. Based on these promising results DLR further matured its high-fidelity approach and currently is able to predict the motion of simplified 6-DOF, 7-DOF and 8-DOF cargo and cargo-parachute systems within the vortical wake of the aircraft. The methodology, the verification and validation of this approach is presented in the following.

\section{Methodology of the Simulation Approach}

The simulation environment AIRBORNE was established in coupling the DLR TAU flow solver and the commercial multi-body simulation software SIMPACK. While TAU computes the aerodynamic forces and moments on the bodies SIMPACK integrates the flight mechanic equations of motion under consideration of kinematic constraints. Details about the investigated airdrop configurations, both software tools and the coupling process are given in the following.

\section{II.A. Description of Airdrop Configurations}

The airdrop simulations were carried out in the wake of a future military transport aircraft (FMTA). The CFD geometry of the FMTA is shown in figure 1. It consists of the fuselage with open ramp, landing gear fairings, wings, propeller pylons, flap track fairings and a T-tail. The rear fuselage is hollow to represent the cargo compartment out of which the cargo configurations are ejected. The wind tunnel model of the FMTA additionally houses a mechanical airdrop mechanism in the cargo compartment which served as ejection mechanism. ${ }^{20}$ The 1:21 scale wind tunnel model of the FMTA has a wing span of approx. $2000 \mathrm{~mm}$.

The investigated airdrop configurations always consist of a simplified payload, represented by a blunt, cuboid cargo body as shown in figure 2. The dimensions of the payload in 1:21 scale are $110 \mathrm{~mm} \times 50 \mathrm{~mm} \times$ $90 \mathrm{~mm}$ (length $\mathrm{x}$ height $\mathrm{x}$ width) and remained constant throughout the work. Its mass, however, changes between the configurations as several payload versions had been manufactured for the experiments, using different materials. Thus, the influence of the mass per unit area on the trajectory could be examined. In

a Abbrev. of: Military Transport Aircraft, project durations 2006-2009 and 2010-2014 


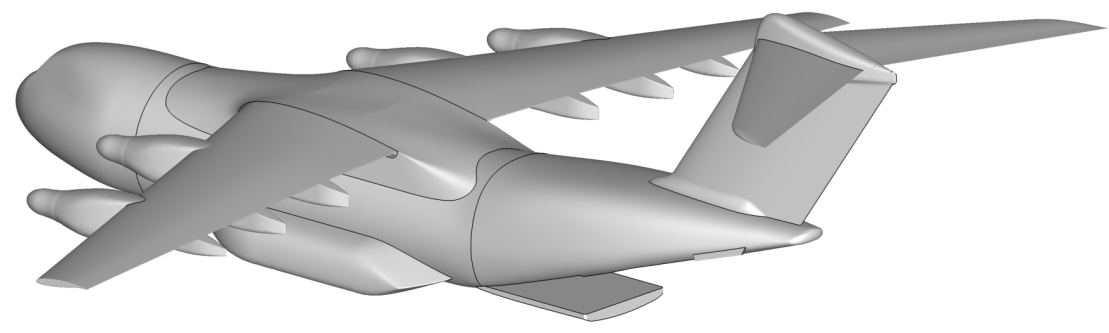

Figure 1. CFD geometry of the FMTA.

1:21 scale it ranges from $62 \mathrm{~g}$ up to $550 \mathrm{~g}$. Applying the relevant scaling laws for trajectory similarity, as for example given in Ref. 21, the corresponding full scale masses are in the range of $575 \mathrm{~kg}-5100 \mathrm{~kg}$.

To gradually increase the complexity of the airdrop configuration several types of parachute models could be connected to the payload. According to figure 2 they are denoted PO, P1 and P2 in the following. They differ in their geometrical representation and in the way they are linked to the payload. While models $P 0$ and $P 1$ consist of a hemispherical shell of constant thickness and a single apex vent hole only, model $P 2$ is based on a real ring-slotted extraction parachute. In the following, the configurations with parachute models P1 and P2, denoted Cargo $+P 1$ and Cargo $+P 2$, will be discussed only. The results for configuration Cargo $+P 0$ were published in 2011 already. ${ }^{14}$ The configuration without parachute served for verification purposes only. Hence, a more detailed insight into configurations Cargo $+P 1$ and Cargo $+P 2$ is given in the following.

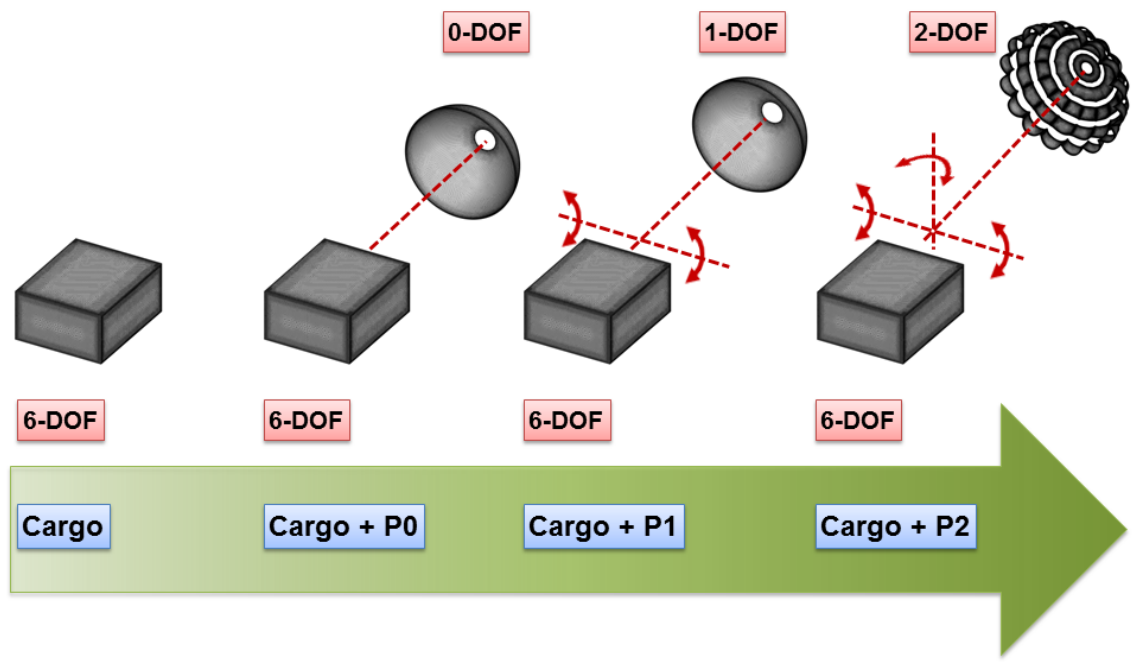

Figure 2. Evolution of the simulated configurations towards more realistic airdrop configurations.

\section{II.A.1. Configuration Cargo+P1}

In this configuration the parachute $P 1$ is hinged to the payload. The hinge line is illustrated by the red, dashed line in figure 2. It intersects the prolonged symmetry axis of the payload at a point located $13.6 \%$ of the cargo length $L$ behind its rear face. The hinge allows the parachute to perform a relative 1-DOF pitching motion in the vertical symmetry plane of the payload. The distance between the parachute inlet plane and the hinge point always remains constant, i.e. the parachute is moving on an arc segment with a radius of $4.2 \times L$ around the hinge point. If the relative pitch angle becomes zero the parachute symmetry axis is aligned with the prolonged symmetry axis of the payload. The parachute itself is represented by the aforementioned simplified model of an inflated extraction parachute. The shell thickness is $0.9 \% \times L$, while the outer diameter amounts to $1.45 \times \mathrm{L}$. The geometric porosity of $P 1$ amounts to $3.5 \%$ only. 
Figure 3(a) depicts the initial position and attitude, as well as the most important geometric parameters, of configuration Cargo $+P 1$ behind the FMTA.

\section{II.A.2. Configuration Cargo+P2}

Compared to the aforementioned configuration the complexity of this configuration is augmented in two ways. First, the rigid hemisphere was substituted by a scaled-down model of a 15-feet ring-slotted and fully inflated drogue parachute. The outer diameter of $P 2$ is $2 \times L$, i.e. its nominal inlet area is increased by nearly $90 \%$ compared to $P 1$. Its geometric porosity amounts to $18 \%$, representing a significant step towards more realistic airdrop configurations. The constant shell thickness is reduced to $0.45 \% \mathrm{x} L$. Apart from the geometric differences P2 is free to perform a 2-DOF relative pitching and yawing motion behind the payload. Thus, the motion of $P 2$ is now taking place on the shell of a virtual sphere whose origin is coincident with the hinge point. The latter is still located at $13.6 \% \times L$ behind the rear face of the payload as before. If the relative pitch and yaw angles become zero P2 is fully aligned with the symmetry axis of the payload. The distance between payload and parachute is increased to $7.3 \times L$, i.e. the parachute is located nearly $75 \%$ further aft of the payload, which better complies with real airdrop scenarios. The initial position and attitude, as well as the most important geometric parameters, of configuration Cargo $+P 2$ behind the FMTA are shown in figure $3(\mathrm{~b})$.

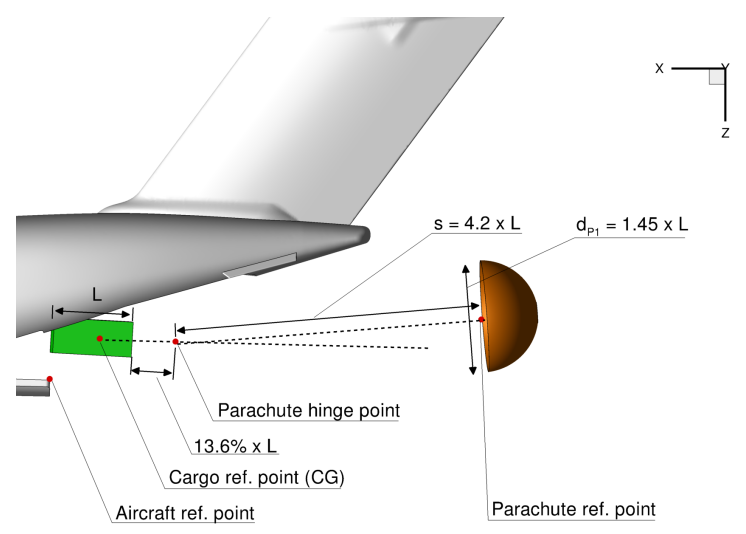

(a) $C a r g o+P 1$

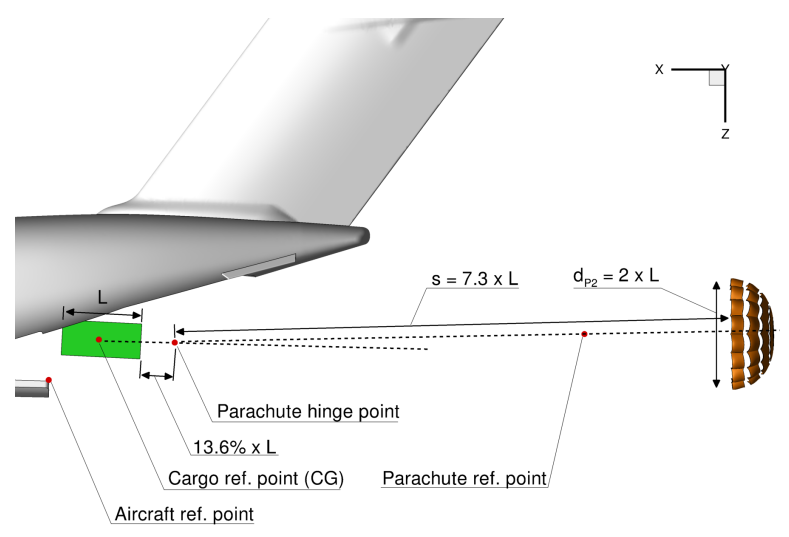

(b) $C a r g o+P 2$

Figure 3. Overview on the initial position and attitude of the airdrop configurations.

\section{II.B. Grid Generation and Grid Assembly}

The grids were generated using the hybrid mesh generator CENTAUR, a commercial software developed by CentaurSoft. ${ }^{22}$ The grid of each body was generated separately in such a way to yield a non-dimensional first wall spacing of $y^{+} \approx 1$. It was thus ensured that the viscous effects in the boundary layer were properly resolved. To achieve this, the local Reynolds number was computed for each body, based on its characteristic length. While the mean aerodynamic chord was used as reference length for the FMTA the projected height perpendicular to the onflow velocity was chosen for the main body and the parachutes. The volume mesh, i.e. the cargo compartment and, more importantly, the part where the trajectory was anticipated, was refined to capture the larger vortical flow structures of the aircraft. The same holds for the flow separation areas around the blunt payload and the parachute. Uniform tetrahedral elements with an element size of $1.5 \%$ of the mean aerodynamic chord were applied to achieve a high spatial resolution in these regions. Simultaneously, the mesh in the trajectory region is very homogeneous, which makes the application of the overset grid technique easier. The latter technique is applied to simulate the relative motion of these bodies in CFD. An overview on the resulting FMTA mesh including refinement regions is shown in figure 4. During mesh generation, the symmetry characteristics of each body were utilized, i.e. only one half of the aircraft was meshed and subsequently mirrored. Main body and parachutes were meshed as quarter-models and mirrored twice in the following. Hence, the symmetry of the computational domain was ensured. Table 1 gives an overview on the resulting node numbers of the component meshes. 


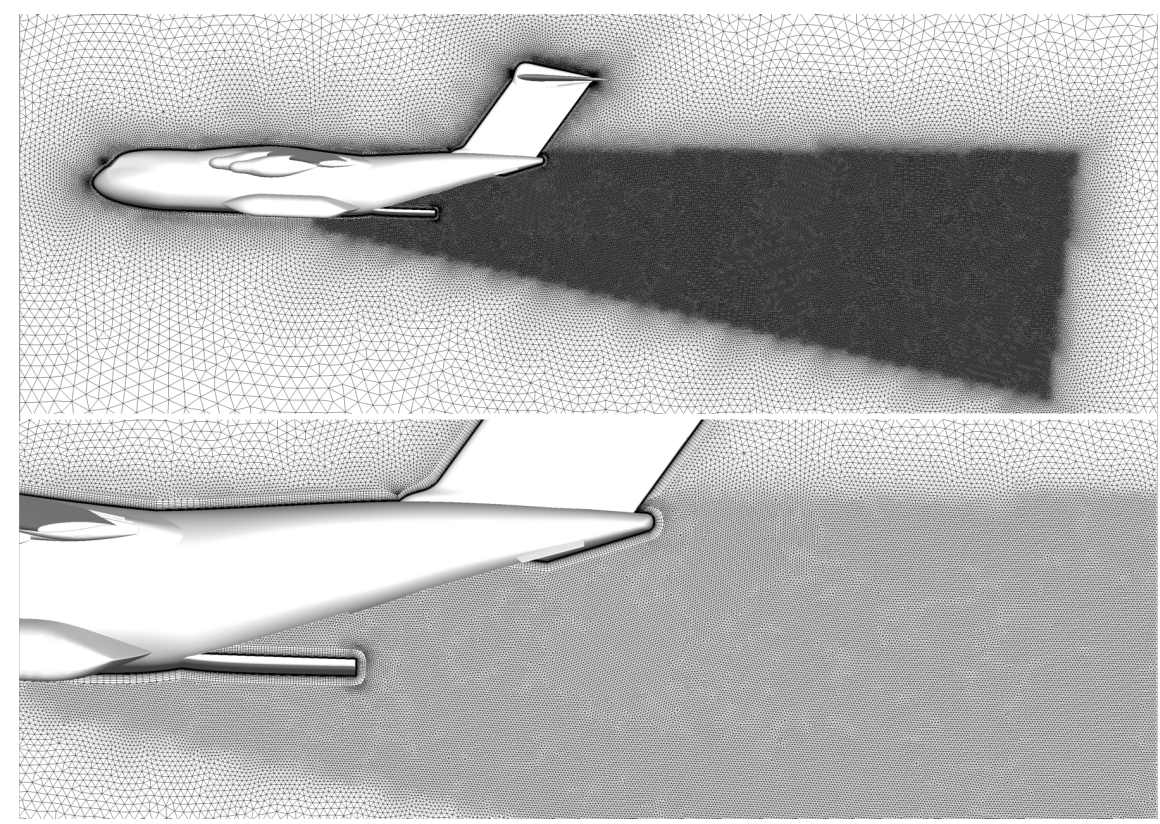

Figure 4. Global and magnified view on the volume mesh at the symmetry plane of the FMTA.

In contrast to the experiments, the mechanical elements connecting the parachute to the payload have not been considered in the CFD geometry to save grid points.

Based on the component grids the final full-configuration grid for the airdrop simulation was obtained in applying the overset grid functionalities ${ }^{23,24}$ of the DLR TAU code. TAU provides two options to use overset grids. The first option consists in using pre-defined holes in the background grid, i.e. the FMTA grid in our case. The grid of the child component, for example the payload grid, is then superimposed. While this approach is rather straightforward and convenient when only small, well-known motions are to be simulated such as the deflection of a control surface it is not suitable for simulating airdrop trajectories. The reason consists in the fact that the exact trajectory and, as a consequence, the location and the dimensions of the required hole are not known in advance.

Therefore, the second option was chosen which makes use of a semi-automatic hole cutting process. The user defines so-called auxiliary hole-definition geometries (HDG's) for every body considered in the simulation in advance. These HDG's are then assigned to their body and rigidly move along with it in the simulation. Thus, the HDG always cuts a hole into the background grid based on the current position and attitude of its assigned body.

To achieve a robust simulation using this technique great attention was paid to the fact that all donor cells in the overlap regions were found. This is particularly important at the very beginning of the airdrop sequence when the airdrop configuration still is very close to the aircraft geometry. Using homogeneous meshes with constant element size in the overlap regions therefore was a crucial step to achieve a robust trajectory simulation.

Table 1. Summary of the resulting component grid sizes.

\begin{tabular}{lc}
\hline \hline Component & approx. \\
\hline Aircraft & $40,500,000$ \\
Cargo & $2,000,000$ \\
Parachute P1 & $1,400,000$ \\
Parachute P2 & $10,500,000$ \\
\hline \hline
\end{tabular}




\section{II.C. The DLR TAU Code}

The computation of the aerodynamic loads within AIRBORNE relies on the DLR TAU code, a hybrid unstructured Reynolds-averaged Navier-Stokes (RANS) solver for three-dimensional, time-accurate and compressible flows. ${ }^{25-27}$ TAU is under continuous development by DLR. Its main developments originated from the German CFD project MEGAFLOW ${ }^{28}$ consolidating contributions from DLR, the aeronautical industry and academia. TAU is an edge-based solver in cell-vertex or cell-centered formulation. A dual-grid approach is used to allow for flow computations on grids being independent of the initial grid cell types. The spatial discretization, based on an upwind scheme or a central scheme with artificial scalar or matrix dissipation, is based on a finite volume formulation. The temporal discretization may either be carried out with an explicit Runge-Kutta or an semi-implicit Lower-Upper Symmetric Gauss-Seidel (LU-SGS) scheme. Several convergence acceleration techniques like residual smoothing or multigrid may be applied. A vast number of turbulence models has been implemented, ranging from algebraic turbulence models up to differential Reynolds stress models. Today, TAU is routinely used in the European aeronautical industry for external aerodynamics.

The spatial discretization for the airdrop simulations relied on a Jameson-type ${ }^{29}$ central scheme with artificial matrix dissipation. A semi-implicit approach was chosen to discretize the time derivative, using a backward difference formula in combination with the LU-SGS scheme as described in Ref. 30. A dualtime stepping approach was employed for the unsteady simulations. The latter were restarted based on a pre-converged steady flow solution. Based on preceding numerical studies (cf. section III) the one-equation turbulence model by Spalart and Allmaras ${ }^{31}$ was applied.

The TAU computations were run on 144 nodes, using the $\mathrm{C}^{2} \mathrm{~A}^{2} \mathrm{~S}^{2} \mathrm{E}^{\mathrm{b}}$ cluster located at the DLR site in Braunschweig, Germany.

\section{II.D. Multi-Body Simulation Software SIMPACK}

The commercial multi-body simulation tool SIMPACK ${ }^{32,33}$ is based on a DLR development. It allows for generating multi-body systems to investigate the dynamic behavior of complex mechanic or mechatronic systems. In the present context SIMPACK was used to integrate the flight mechanic equations of motion for the bodies participating in the airdrop simulation. Its main advantage consists in its ability to easily handle kinematic constraints, such as between the main body and the hinged parachutes in configurations Cargo $+P 1$ and Cargo $+P 2$. The underlying time integration method used in the present work is based on an implicit second-order backward difference formulation (BDF2) algorithm.

\section{II.D.1. Description of the SIMPACK Model}

A 2D-representation of the kinematic model for configuration Cargo+P2 is exemplarily depicted in figure 5 . It consists of five blocks, each one representing a body with kinematic constraints. Apart from the aircraft $\left(\$ B_{-} F M T A\right)$, the payload $\left(\$ B_{-} C_{\text {Cargo }}\right)$ and the parachute $\left(\$ B_{-}\right.$Parachute_P 2$)$, which are also present in the CFD geometry, two auxiliary bodies are additionally used to simulate the relative motion between parachute and cargo. The first auxiliary body $\left(\$ B_{-} J_{o i n t}\right)$ represents the experimental hinge mechanism, which is located just aft of the rear face of the payload as described in section II.A.2. The second auxiliary body (\$B_Rope) represents the rope which connected the parachute to the hinge mechanism in the experiments. Thus, the experimental setup is reproduced as closely as possible from a kinematic point of view. The mass, position of the center of gravity (CG) or the moments of inertia about the CG are then specified by the user for every single body. The same holds for the initial position and attitude of each body.

While SIMPACK offers the capability to consider flexible structures all bodies are assumed to be rigid, as indicated by the respective keyword within each of the five blocks. Hence, structural deformation effects, for instance of the rope or the parachute, are not accounted for. Apart from the definition of the bodies the kinematic dependencies among the bodies are shown in figure 5 , indicated by the blue solid lines and circles. The latter define so-called joints, in which the user specifies the DOF of one body relative to another. The frame surrounding the block diagram represents the inertial reference system. Its coordinate system does not change over time.

Looking at the kinematic relations among the bodies it becomes evident that the FMTA has been modeled as 0-DOF body. It is connected to the inertial reference frame, but to none of the other bodies. The cargo

\footnotetext{
${ }^{\mathrm{b}}$ Center for Computer Applications in Aerospace Sciences and Engineering
} 


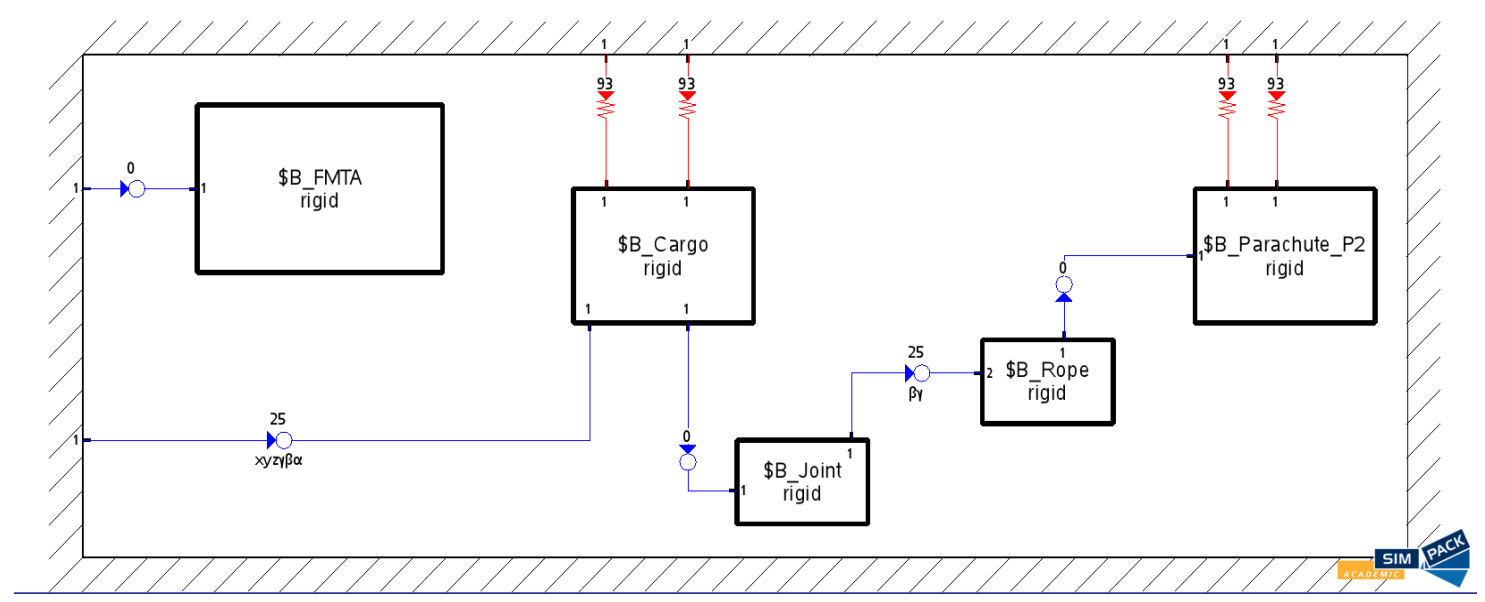

Figure 5. 2D-representation of the SIMPACK model of configuration Cargo + P2.

is also directly linked to the inertial reference frame, however, as a 6-DOF body. The latter is indicated by the literals $(x y z \gamma \beta \alpha)$ below the blue joint symbol in figure 5. Each literal stands for a single degree of freedom. While arabic literals represent the translational degrees of freedom, the greek ones symbolize rotational DOF. Consequently, the cargo features three translational and rotational DOF relative to the inertial reference frame. The hinge mechanism does not have any DOF relative to the cargo, i.e. from a kinematic point of view the cargo and the hinge mechanism are treated as one rigid body. As in the experiments the rope is connected to the hinge mechanism (block $\$ B_{-} J_{o i n t}$ ). To reproduce the real motion of the parachute attached to a single and tensed rope the latter is modeled with two rotational degrees of freedom $(\beta \gamma)$ relative to the hinge mechanism. Finally, the parachute P2 has no additional DOF relative to the rope, i.e. rope and parachute are kinematically treated as one single body again. To summarize, configuration Cargo $+P^{2}$ is represented by a 8 -DOF model in SIMPACK. All other configurations with parachute as depicted in figure 2 basically use the same SIMPACK model. The only distinguishing mark consists in the number of DOF between blocks $\$ B_{-} J o i n t$ and $\$ B_{-}$Rope.

The SIMPACK computations were performed on a desktop workstation at the DLR Institute of Aeroelasticity in Göttingen, Germany.

\section{II.E. Coupling Procedure between TAU and SIMPACK}

The coupling between TAU and SIMPACK is based on a classical co-simulation with dual data exchange within a given time step, based on the conventional serial staggered (CSS) algorithm. ${ }^{34}$ The coupling process is schematically depicted in figure 6 . After TAU has computed the aerodynamic loads they are post-processed first to transform them into a SIMPACK-compatible format, the so-called $\vec{U}$-vector. In the $\vec{U}$-vector information about the three forces and three moments for each body is stored. As TAU and SIMPACK were run on different operating systems and distributed machines the exchange of information between both environments is conducted via a TCP/IP socket. Within SIMPACK the loads contained in the $\vec{U}$-vector are then assigned to the respective bodies, using so-called force elements. They are shown as red springs in figure 5. As the FMTA is not moving in the current version of AIRBORNE the aerodynamic loads acting on it are omitted. Only the loads of the cargo and the parachute are considered in the SIMPACK model and used for time integration.

As soon as SIMPACK has computed the position, attitude angles and the translational and rotational velocities for the next time step the data are stored in the so-called $\vec{Y}$-vector and sent back to an auxiliary tool converting the data into TAU-readable format. The new arrangement of the bodies is performed using the above mentioned overset grid technique and the CFD computation starts again.

The coupled unsteady computations were conducted with a time step size equivalent to $600 \mathrm{~Hz}$, while the experimental data were recorded at $300 \mathrm{~Hz}$. 


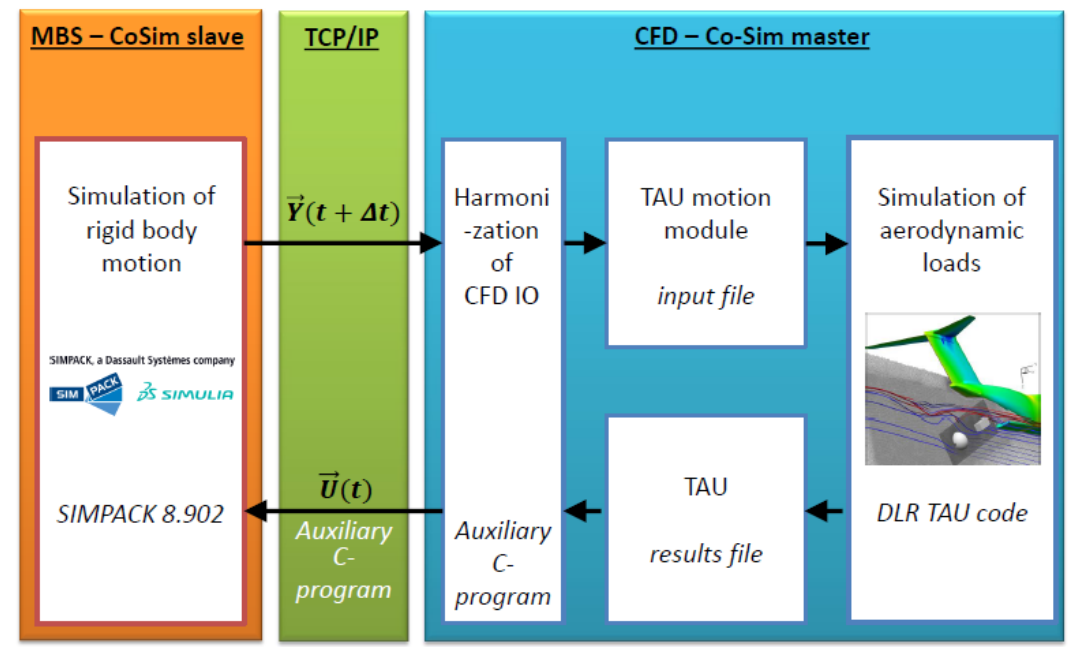

Figure 6. Schematic description of the coupling procedure between TAU and SIMPACK.

\section{Verification of AIRBORNE}

A very important point in the early development of AIRBORNE dealt with the question whether or not RANS methods are suited to capture the vortical flow field behind a military transport aircraft with open ramp. A literature survey revealed that other researchers ${ }^{2-4}$ preferably applied delayed detached eddy simulation (DDES) methods to investigate the flow around a similar military transport aircraft or about the canopy of parachutes. Although DDES indisputably resolves more details of the prevailing flow features compared to RANS the question arises whether or not these additional details provide any benefit in terms of accuracy when it comes to trajectory computations. Even if that is the case the gain in accuracy needs to be sufficiently high to legitimate the higher computational costs in applying DDES. To the knowledge of the authors no investigations addressing the impact of the numerical method on the accuracy of a trajectory have been published yet.

As a consequence DLR carried out its own study ${ }^{14}$ on the impact of each of the two numerical methods in predicting the typical flow features of an aircraft with open ramp. At the low experimental Reynolds number in the range of 300,000 - 400,000, based on the reference chord length of the FMTA, DLR found no difference between DDES and unsteady RANS results compared to experimental data. Hence, the development of AIRBORNE was fostered with the more time-efficient RANS methods.

The impact of the turbulence in predicting the flow field encountered in airdrop simulations was examined in separate studies. ${ }^{18,19}$ It was shown that the Spalart-Allmaras model revealed the best compromise with respect to accuracy, efficiency and robustness and was therefore used in TAU.

\section{Validation of AIRBORNE and Discussion}

In the following the results of the airdrop simulations of configurations $C a r g o+P 1$ and Cargo $+P 2$ are compared to experimental trajectory data. As the experiments were carried out in 1:21 scale and in ambient wind tunnel conditions the AIRBORNE simulation aimed at reproducing these conditions instead of real airdrop conditions.

\section{IV.A. Accuracy of Experimental Data}

The trajectory of the airdrop configurations in the experiments was recorded with an optical trajectory tracking system, working with a resolution of $1280 \times 1024$ pixels at 300 frames per second. Details on the experimental setup and the calibration procedure can be found in Refs. 11 and 20. The calibration revealed that, within the calibration window, the position of the payload CG can be determined with an accuracy of up to $1 \%$ of its length. In extreme cases, in which the cargo is heavily rotated about all three axes and additionally located outside of the calibration window, the position accuracy in lateral $y$-direction deteriorates to $5 \%$. 


\section{IV.B. Validation for Configuration $\operatorname{Cargo}+P 1$}

\section{IV.B.1. Initial Conditions}

The airdrop experiments for this configuration were conducted at an onflow velocity of $22 \mathrm{~m} / \mathrm{s}$. The FMTA angle of attack is $2^{\circ}$. The Reynolds number amounts to 390,000 , based on the reference chord length of the FMTA, or to 72,000, when based on the height of the cargo. The evaluation of the experimental data revealed slightly asymmetric initial conditions for this configuration, i.e. all three Euler angles of the cargo are of non-zero values. In particular the following initial angles were used in the simulation: yaw angle of $-2.4^{\circ}$, pitch angle of $3.3^{\circ}$ and roll angle of $-0.4^{\circ}$. Figure $3(\mathrm{a})$ shows the side view of the initial situation. Apart from the pitch angle the initial yaw angle of the payload can be recognized as well as its front face is visible in the side view. While small initial yaw and roll angles existing in the experiments always had been neglected in the simulations so far, it was decided here to conduct the simulation without that simplification. Hence, the present initial conditions were considered to be the most challenging ones of all simulated configurations. In addition, the parachute is not aligned with the longitudinal axis of the main body as shown in figure 3(a). Instead, it is deflected in negative $z$-direction, with a relative pitch angle of $-7.4^{\circ}$ compared to the longitudinal axis of the cargo. The mass of the cargo amounts to $520 \mathrm{~g}$, whereas the parachute mass is $39 \mathrm{~g}$. As stated earlier, the motion of configuration Cargo $+P 1$ is computed using a 7-DOF model.

\section{IV.B.2. Results and Discussion}

Figure 7 shows the simulated trajectory of the cargo compared to experimental data. For the sake of clarity only every fourth experimental data point is plotted. The position of the CG is plotted in terms of translational displacement in all three spatial directions over time. As seen in figure 7(a) the agreement in predicting the longitudinal motion in $x$ - and $z$-directions is very good as the simulated trajectory runs closely to the mean value of the experimental data. Due to the asymmetric initial conditions there is also a motion in lateral $y$-direction, towards the starboard wing of the aircraft. While the simulation reproduces this behavior correctly in qualitative terms it underestimates the absolute displacement of the CG in that direction. According to the mean value of the experimental data the CG of the cargo moves about $160 \mathrm{~mm}$, or 1.45 times its length $L$, in lateral direction. In the simulation, however, the distance traveled amounts to a mere $40 \mathrm{~mm}$, or $0.36 \times L$, only. As will be discussed in the following the reason for these differences is probably related to deviations in the yawing motion of the cargo.

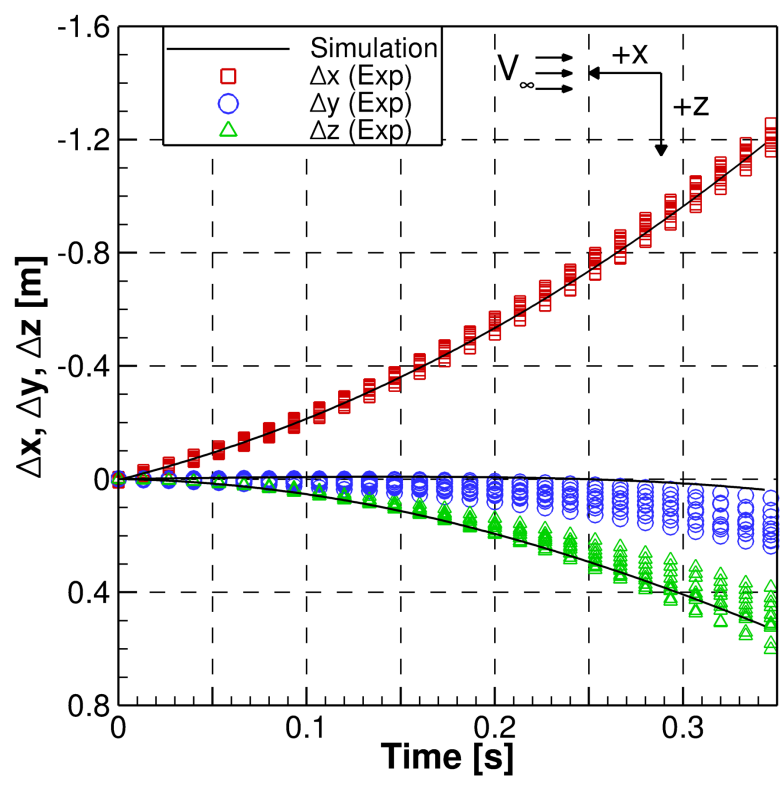

(a) Translation of the CG

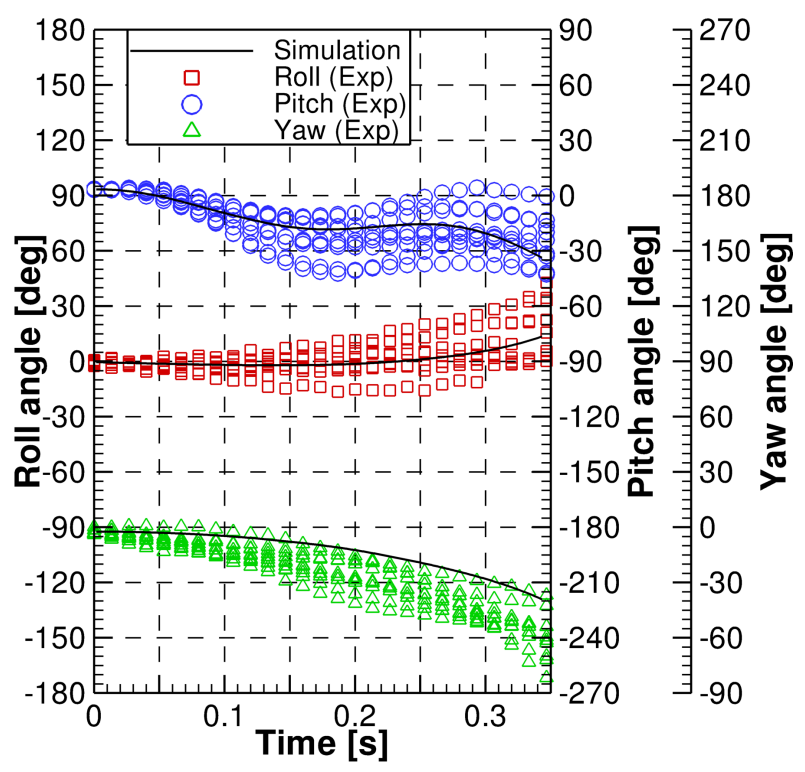

(b) Rotation

Figure 7. Comparison of the payload trajectory for configuration Cargo $+P 1$.

The attitude of the cargo during airdrop is shown in figure $7(\mathrm{~b})$ in terms of its Euler angles. Again, only every fourth experimental data point is plotted. Due to the asymmetric initial conditions the payload 
rotates about all three axes during its drop, exhibiting a dominant pitching and yawing motion. The pitching motion can be subdivided into three characteristic phases: (1) until $t=0.15 \mathrm{~s}$ the pitch angle is continuously reducing to values of $\Theta \approx-18^{\circ}$, while in phase (2) between $0.15 \mathrm{~s}<t<0.25 \mathrm{~s}$ it nearly remains constant, before it further reduces in phase (3) for $t>0.25 \mathrm{~s}$. As shown the simulation was able to correctly predict this characteristic behavior both in qualitative and quantitative terms.

The yawing motion of the cargo is also well captured in qualitative terms, however it is underestimated quantitatively by the simulation. Although the simulated yaw angle largely is within the spread of the experimental data it becomes evident that its gradient, i.e. the yaw velocity starts deteriorating in the first $0.15 \mathrm{~s}$ already. The reason for this is most probably attributed to differences in the initial conditions of this configuration. As stated above the deficiencies occurring in the yawing motion also affect the translation in lateral direction in the following way: with increasing yaw angle of the cargo the parachute behind the cargo is also deflected to a greater extent into the free stream air, thus pulling the cargo further into lateral direction.

The roll angle largely remains very small, until after $0.25 \mathrm{~s}$ a more pronounced rolling motion sets in, which is well predicted by the simulation.

The resulting parachute motion is illustrated in figure 8. The characteristic cosine-type pitching motion of the parachute is well predicted by the simulation. The frequency of the parachute oscillation of $3.6 \mathrm{~Hz}$ is perfectly matched. It can be noticed, though, that the experiments reveal a slightly delayed parachute oscillation compared to the simulation. This phase shift is most probably attributed to the initial conditions of the parachute, in particular its initial pitching velocity. As can be observed in the first $0.03 \mathrm{~s}$ the experimental data points consistently suggest a further reduction of the relative pitch angle towards more negative values. The simulation, in contrast, predicts an increase nearly from the beginning. A further study on the initial pitch velocity of the parachute will therefore be conducted to support this assumption.

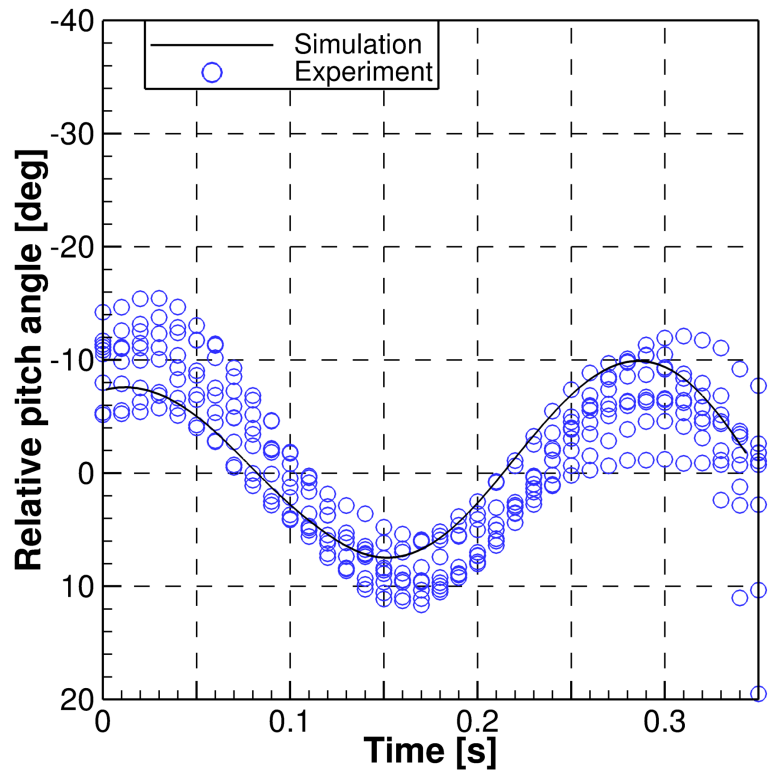

Figure 8. Comparison of the parachute motion at configuration Cargo $+P 1$.

\section{IV.C. Configuration Cargo+P2}

\section{IV.C.1. Initial Conditions}

The experiments for configuration Cargo $+P 2$ were also carried out at an FMTA angle of attack of $2^{\circ}$. The onflow velocity was reduced to $18 \mathrm{~m} / \mathrm{s}$. The resulting Reynolds numbers amount to 318,000 or 59,000, based on the aforementioned reference lengths. A side view on the initial position and attitude of this configuration is shown in figure 3(b). In contrast to configuration $C a r g o+P 1$ this configuration was subject to symmetric initial conditions, i.e. the initial roll and yaw angles are zero in the simulation. The initial pitch angle was set to $3.0^{\circ}$. The parachute is again deflected into upward direction, with a relative pitch angle of $-3.8^{\circ}$. The 
second DOF of the parachute, its relative yawing angle, initially is zero. The mass of the cargo amounts to $335 \mathrm{~g}$, whereas the parachute mass is $7.8 \mathrm{~g}$. The motion of configuration Cargo $+P^{2}$ is computed using an 8-DOF model.

\section{IV.C.2. Results and Discussion}

Figure 9 compares the trajectory of the cargo between simulation and experiments. Again, only every fourth experimental data point is plotted for the sake of clarity. The qualitative agreement in predicting the motion of the cargo CG is excellent as shown in figure 9(a). The gradients are mostly well captured and, for most of the time, the agreement is also very good in quantitative terms. Differences occur towards the end of the simulation for the translation in $x$-direction, where the simulation overpredicts the motion, indicating that the configuration is moving faster away from the aircraft in downstream direction than in the experiments. At the end of the simulation after $0.32 \mathrm{~s}$, for example, the CG has traveled $140 \mathrm{~mm}$ further into downstream direction than the experimental mean value suggests. This corresponds to $1.3 \times 2$. The reason for this difference is not yet fully understood. However, as the differences accumulate over time they are probably attributed to the initial settings of the time-accurate simulation as well. Regarding the $y$-direction no motion takes place due to the symmetric initial conditions. In $z$-direction a similar offset as in $x$-direction accumulates over time, however, less pronounced. In the simulation the cargo-parachute configuration is falling faster than in the experiments. The reason for this overprediction is probably originating from the parachute motion, as will be discussed below.

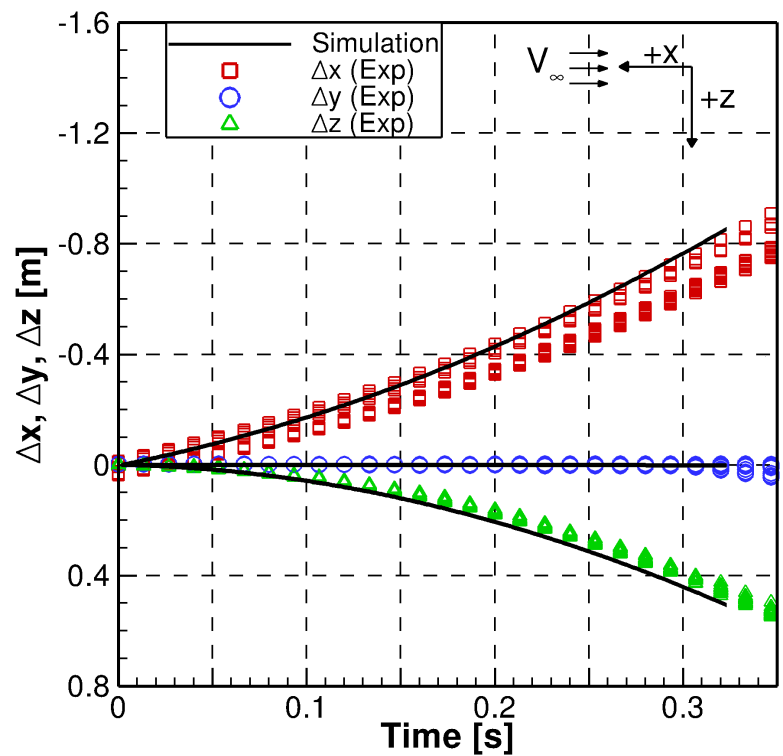

(a) Translation of the CG

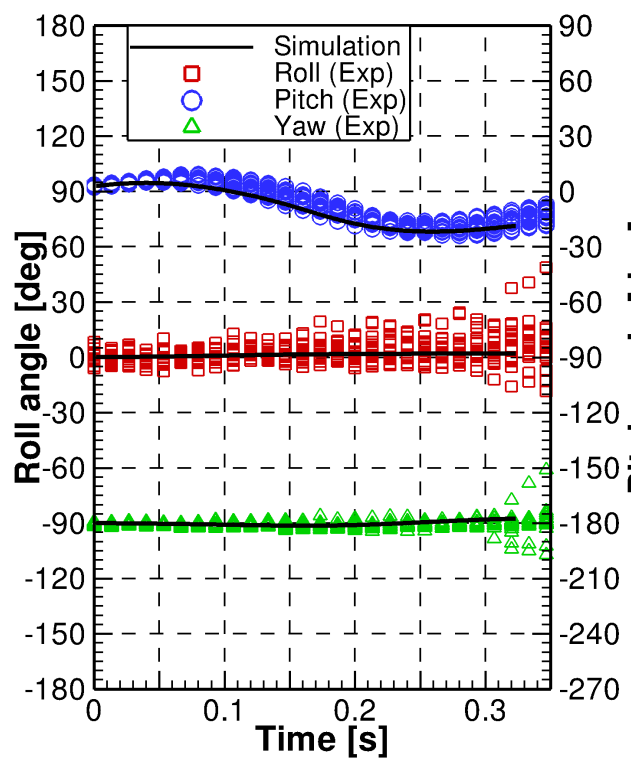

(b) Rotation

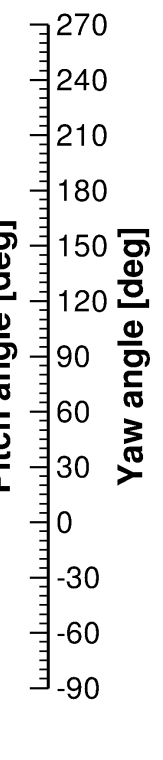

Figure 9. Comparison of the payload trajectory for configuration Cargo+P2.

The rotation of the payload is depicted in figure 9(b). A significant motion only takes place about the pitch axis. The characteristic pitching motion was correctly captured, the simulation curve always lies fully within the experimental data points. Moreover, when comparing the pitch angle curves for both configurations as shown in figures 7(b) and 9(b) it becomes evident that the reproduceability in the experiments is higher in case of configuration Cargo $+P 2$, as the bandwidth of the experimental data is much narrower. This is attributed to the lower weight and the more stable alignment of parachute $P 2$ behind the payload due to its higher geometric porosity and due to the fact that it can better react to local flow disturbances as it is connected with a rope to the payload, which increases its degrees of freedom. Thus, potential aerodynamic instabilities at the parachute do not translate that much into an attitude change of the payload.

The motion of the parachute is plotted in figure 10. Again, the qualitative agreement is considered very good. Quantitatively, the simulated relative yaw angle matches the experimental results very well. However, although a 2-DOF model has been used to simulate the parachute its pitching motion again is dominant. Although the simulation always lies within the experimental spread for this parameter it becomes evident 


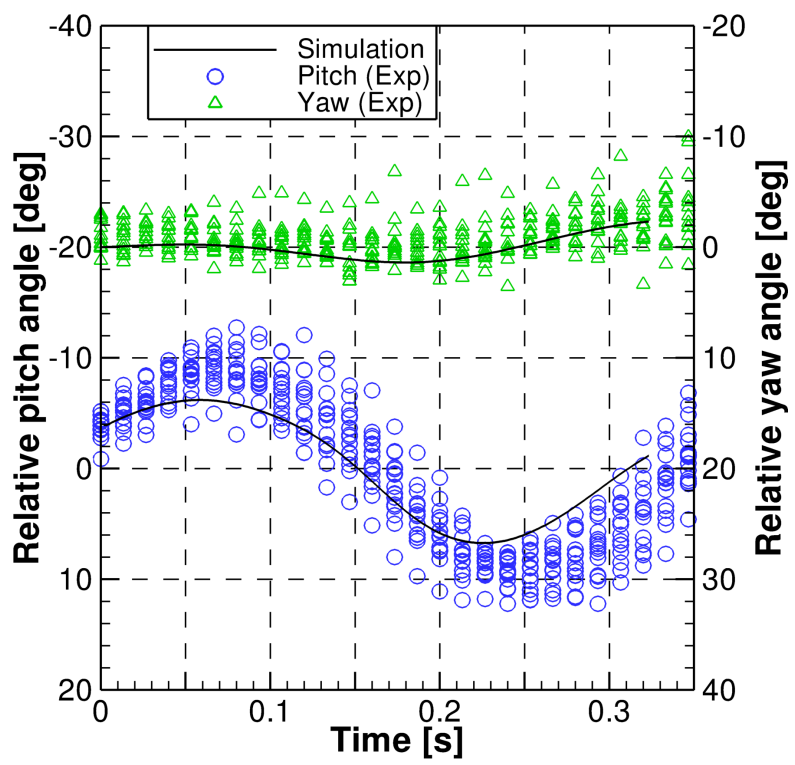

Figure 10. Comparison of the parachute motion at configuration Cargo+P2.

that the mean oscillation magnitude is underestimated by $3^{\circ}-4^{\circ}$. The oscillation frequency of $2.9 \mathrm{~Hz}$ is correctly predicted. A similar phase shift as seen at configuration Cargo $+P 1$ is visible again, most probably for the same reasons, i.e. a too low initial pitch velocity. This assumption is supported by the gradient of the simulated curve within the first $0.1 \mathrm{~s}$. While the experimental data approach a local minimum in relative pitch angle in the range of $-8^{\circ}$ to $-14^{\circ}$ after $0.08 \mathrm{~s}$ with a nearly constant gradient, the gradient is continuously reducing in the simulation right from the beginning. After $0.05 \mathrm{~s}$ a sign reversal in the gradient of the simulated curve can be observed which is not present that early in the experimental data. Thus, the impact of the initial parachute pitch velocity needs to be assessed further to improve the accuracy. The resulting trajectory of configuration Cargo $+P^{2}$ is exemplarily shown for three time instances in figure 11.

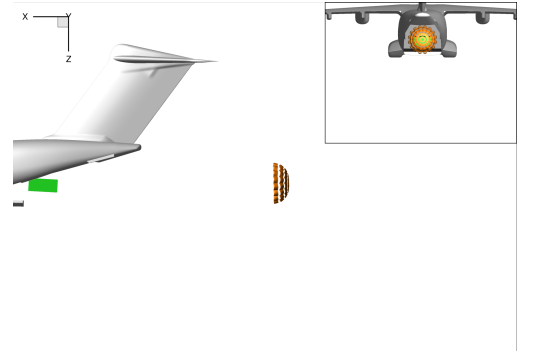

(a) $t=2 \mathrm{~ms}$

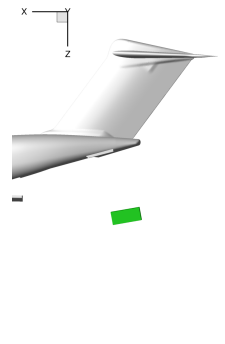

(b) $t=162 \mathrm{~ms}$
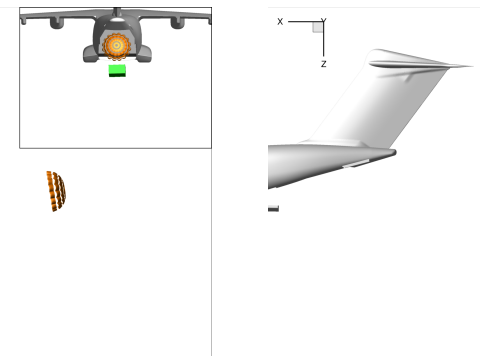

(c) $t=323 \mathrm{~ms}$

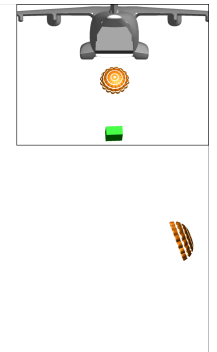

Figure 11. Visualization of the simulated trajectory of configuration Cargo $+P 2$ at the beginning (left), at an intermediate stage (middle) and at the end of the simulation (right).

\section{Conclusion}

In the present paper an airdrop simulation tool called AIRBORNE is presented. AIRBORNE belongs to a multi-fidelity simulation approach developed by DLR to evaluate and assess airdrop operations for future military transport aircraft. The different tools of this multi-fidelity approach enable DLR to examine airdrop operations with tools that are specifically suited for the different phases of an airdrop. Out of these tools AIRBORNE has been developed specifically to investigate the aerodynamic interaction between the payload and the flow field behind the aircraft in the initial phase of an airdrop. Airdrop failures are often related to problems occurring in that initial phase. With the help of AIRBORNE DLR is able to compute the initial trajectory of up to 8-DOF cargo-parachute configurations in the wake of the aircraft. Thus, potentially 
hazardous situations can be prematurely identified and critical conditions, e. g. an asymmetric insertion of the payload into the flow field, can be examined.

The validation of AIRBORNE is currently based on wind tunnel airdrop experiments. Thus, in a next step the Reynolds number effects on the trajectories need to be investigated. The validation for both configurations presented in this paper revealed a very good agreement in qualitative terms. Even in quantitative terms the simulation results largely are within the bandwidth of the experimental data. Nevertheless, for some parameters quantitative differences were identified towards the end of the simulation. The authors believe that these are foremost traced back to inaccuracies in the initial conditions of the unsteady simulation. The general methodology of AIRBORNE, in contrast, is believed to be reasonable and well-suited to account for the relevant flow interference effects during airdrop, especially when keeping in mind that, from an aerodynamic point of view, the simulated flow conditions cannot be considered but challenging.

\section{Acknowledgments}

The authors would like to thank N. Bier from the Transport Aircraft Department of the DLR Institute of Aerodynamics and Flow Technology and the entire team of the DNW-NWB for their support in preparing, conducting and analyzing the wind tunnel airdrop experiments. Furthermore, the authors would like to thank A. Raichle and G. Einarsson from the $\mathrm{C}^{2} \mathrm{~A}^{2} \mathrm{~S}^{2} \mathrm{E}$ department of the DLR Institute of Aerodynamics and Flow Technology as well as J. Arnold of the DLR Institute of Aeroelasticity for their continuous support in establishing the coupled simulation process.

\section{References}

\footnotetext{
${ }^{1}$ Benney, R., Krainski, W., Onckelinx, P., Delwarde, C., Mueller, L., and Vallance, M., "NATO Precision Airdrop Initiatives and Modeling and Simulations Needs," Fluid Dynamics of Personnel and Equipment Precision Delivery from Military Platforms, Meeting Proceedings RTO-MP-AVT-133, Keynote 2, pp. KN2-1-KN2-22, 2006.

${ }^{2}$ Serrano, M., Leigh, E., III, W. J., Forsythe, J., Morton, S., and Squires, K., "Computational Aerodynamics of the C-130 in Airdrop Configuration," AIAA paper 2003-229, January 2003.

${ }^{3}$ Morton, S., Tomaro, R., and Noack, R., "An Overset Unstructured Grid Methodology Applied to a C-130 with a Cargo Pallet and Extraction Parachute," AIAA paper 2006-0461, January 2006.

${ }^{4}$ Bergeron, K., Cassez, J.-F., and Bury, Y., "Computational Investigation of the Upsweep Flow Field for a Simplified C-130 Shape," AIAA paper 2009-90, January 2009.

${ }^{5}$ Pang, S., Ng, E., and Chiu, W., "Comparison of Turbulence Models in Near Wake of Transport Plane C-130H Fuselage," Journal of Aircraft, Vol. 50, No. 3, 2013, pp. 847-852.

${ }^{6}$ Desabrais, K., "The Motion and Aerodynamics of an Airdrop Platform," AIAA paper 2004-4845, August 2004.

${ }^{7}$ Desabrais, K., "Aerodynamic Forces on an Airdrop Platform," AIAA paper 2005-1634, May 2005.

${ }^{8}$ McQuilling, M., Potvin, J., and Riley, J., "Simulating the Flows About Cargo Containers Used During Parachute Airdrop Operations," Journal of Aircraft, Vol. 48, No. 4, 2011, pp. 1405-1411.

${ }^{9}$ Murman, S., Aftosmis, M., and Berger, M., "Simulations of Store Separation from an F/A-18 with a Cartesian Method," Journal of Aircraft, Vol. 41, No. 4, 2004, pp. 870-878.

${ }^{10}$ Panagiotopoulos, E. and Kyparissis, S., "CFD Transonic Store Separation Trajectory Predictions with Comparison to Wind Tunnel Investigations," International Journal of Engineering, Vol. 3, No. 6, 2010, pp. 538-553.

${ }^{11}$ Jann, T., Geisbauer, S., Bier, N., Krüger, W.-R., and Schmidt, H., "Multi-Fidelity Simulation of Cargo Airdrop: From the Payload Bay to the Ground," AIAA paper submitted to the AIAA Modeling and Simulation Technologies Conference, June 2015.

12 Jann, T., "Coupled Simulation of Cargo Airdrop from a Generic Military Transport Aircraft," AIAA paper 2011-2566, May 2011.

${ }^{13}$ Jann, T. and Geisbauer, S., "Approximated Steady Aerodynamic Characteristics for two Cuboids and a Hemispherical Shell used in Airdrop Simulation," AIAA paper 2013-1282, March 2013.

${ }^{14}$ Geisbauer, S., Schade, N., Enk, S., Schmidt, H., and Arnold, J., "Experimental and Numerical Investigation of the Flow Topology During Airdrop Operations," AIAA paper 2011-2565, May 2011.

${ }^{15}$ Roosenboom, E., Schröder, A., Agocs, J., and Geisler, R., "Experimental Investigation of the Flow Field Topology for Several Cargo Drop Configurations," AIAA paper 2012-3198, June 2012.

${ }^{16}$ Roosenboom, E., Schröder, A., Agocs, J., and Geisler, R., "Coherent Wake Structures for Transport Aircraft at Cargo Airdrop Configurations Including Parachutes," AIAA paper 2013-2536, June 2013.

${ }^{17}$ Löser, T. and Bergmann, A., "Capabilities of Deployment Tests at DNW-NWB," In Fluid Dynamics of Personnel and Equipment Precision Delivery from Military Platforms, Meeting Proceedings RTO-MP-AVT-133, Paper 13, pp. 13-1-13-11, 2006.

${ }^{18}$ Roosenboom, E., Schade, N., Acisu, I., and Schröder, A., "Experimental and Numerical Investigation of the Near-Field Wake behind a Transport Aircraft Configuration with an Open Ramp," Paper, $4^{\text {th }}$ Symposium on Integrating CFD and Experiments in Aerodynamics, September 2009.
} 
${ }^{19}$ Geisbauer, S., Bier, N., Kirz, J., and Roosenboom, E., "Validation of the Flow Topology Around Several Airdrop Cargo Configurations at Static Conditions," AIAA paper 2013-3155, June 2013.

${ }^{20}$ Schade, N., "Simulation of Trajectories of Cuboid Cargos Released from a Generic Transport Aircraft," AIAA paper 2011-3959, June 2011.

${ }^{21}$ Wolowicz, C., Bowman, Jr., J., and Gilbert, W., "Similitude Requirements and Scaling Relationships as Applied to Model Testing," NASA Technical Paper 1435, August 1979.

${ }^{22}$ CentaurSoft, "Homepage," http://www.centaursoft.com, retrieved May 2015.

${ }^{23}$ Madrane, A., Heinrich, R., and Gerhold, T., "Implementation of the Chimera Method in the Unstructured Hybrid DLR Finite Volume TAU-Code," Paper, $6^{\text {th }}$ Overset Composite Grid and Solution Technology Symposium, October 2002.

${ }^{24}$ Madrane, A., Raichle, A., and Stürmer, A., "Parallel Implementation of a Dynamic Overset Unstructured Grid Approach," Proceedings of the Third International Conference on Computational Fluid Dynamics, ICCFD3, European Community on Computational Methods in Applied Sciences, 2004.

${ }^{25}$ Gerhold, T., Galle, M., Friedrich, O., and Evans, J., "Calculation of Complex Three-Dimensional Configurations Employing the DLR-Tau Code," AIAA paper 97-0167, January 1997.

${ }^{26}$ Gerhold, T., "Overview of the Hybrid RANS Code TAU," MEGAFLOW - Numerical Flow Simulation for Aircraft Design, edited by N. Kroll and J. Fassbender, Vol. 89 of Notes on Numerical Fluid Mechanics and Multidisciplinary Design, Springer, 2005, pp. 81-92.

${ }^{27}$ Schwamborn, D., Gerhold, T., and Heinrich, R., "The DLR TAU-Code: Recent Applications in Research and Industry," In Proceedings of the European Conference on Computational Fluid Dynamics, ECCOMAS, CFD 2006, edited by P. Wesseling, E. Oñate, and J. Périaux, 2006.

${ }^{28}$ Kroll, N., Rossow, C.-C., Becker, K., and Thiele, F., "MEGAFLOW - A Numerical Flow Simulation System," Aerospace Science and Technology, Vol. 4, No. 4, 2000, pp. 223-237.

${ }^{29}$ Jameson, A., Schmidt, W., and Turkel, E., "Numerical Solution of the Euler Equations by Finite Volume Methods Using Runge-Kutta Time Stepping Schemes," AIAA paper 81-1259, June 1981.

${ }^{30}$ Dwight, R., "Efficiency Improvements of RANS-based Analysis and Optimization Using Implicit and Adjoint Methods on Unstructured Grids," Ph.d. thesis, university of manchester, 2006.

${ }^{31}$ Spalart, P. and Allmaras, S., "A One-Equation Turbulence Model for Aerodynamic Flows," AIAA paper 1992-439, January 1992.

${ }^{32}$ SIMPACK, "Homepage of the SIMPACK AG," http://www.simpack.com, retrieved May 2015.

${ }^{33}$ Krüger, W.-R., Heinrich, R., and Spieck, M., "Fluid-Structure Coupling Using CFD and Multibody Simulation Methods," Paper ICAS 2002-4.6.2, September 2002.

${ }^{34}$ Farhat, C. and Lesoinne, M., "Higher-Order Staggered and Subiteration Free Algorithms for Coupled Dynamic Aeroelasticity Problems," AIAA paper 98-0516, January 1998. 